\title{
Constraint Programming
}

\author{
Pascal Van Hentenryck \\ Brown University, Providence RI 02912, USA
}

Constraint programming is a remarkable success story. It quickly moved from research laboratories to industrial applications in the late 1980s and is in daily use to solve complex optimization throughout the world. At the same time, constraint programming has continued to evolve, addressing new needs and opportunities. This talk reviews some recent progress in constraint programming. The first part of the talk starts with its fundamental contribution, the ability to express and exploit combinatorial substructures to prune infeasible solutions and find feasible solutions. It also reviews some of the benefits of its architecture on a variety of applications in scheduling, rostering, and combinatorial matching, emphasizing the underlying modeling and computation techniques. The second part of the talk argues that constraint programming is an integration technology and reviews some hybridizations of constraint programming, including local search, mathematical programming, and global optimization. The final part of the talk gives a brief overview of some promising, novel applications of constraint programming.

Acknowledgments. This work was supported in part by the U.S. Department of Homeland Security's National Infrastructure Analysis and Simulation Center (NISAC) Program, by NSF award DMI-0600384, and ONR Award N000140610607. 